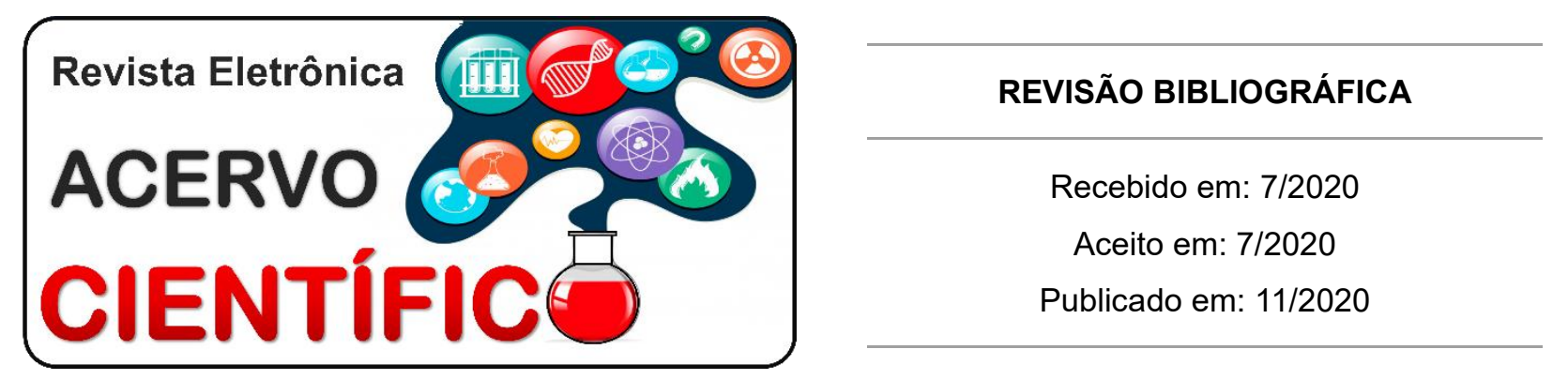

\title{
Bloqueio peridural no controle da dor crônica
}

\author{
Epidural block in chronic pain control
}

\section{Bloqueo peridural en el control del dolor crónico}

Adriana Simões Ferreira ${ }^{1 *}$, Diego de Matos Silveira², Ana Luíza Ferreira de Azevedo ${ }^{3}$, Aquila Marcelle Dias Ferreira ${ }^{4}$, Camila Ferreira Mariani ${ }^{5}$, Caroline Bergeier ${ }^{6}$, Douglas Almeida Costa ${ }^{7}$, Gabriela Horst Dutra Coutinho ${ }^{8}$, Kamille Lírio Ramos ${ }^{5}$, Rafaelle Rocha ${ }^{6}$.

Resumo: Este artigo tem por objetivo apresentar através de uma revisão sobre a eficácia clínica e a segurança do tratamento intervencionista dos bloqueios anestésicos peridurais no controle da dor crônica. A dor crônica é um sintoma recorrente e de difícil controle em diversas entidades clínicas, com mecanismos fisiopatológicos complexos e que gera repercussões sociais, físicas e mentais importantes para a população, além de implicar altos custos tanto para os sistemas de saúde, quanto para a economia. Dentre as formas de intervenção e controle desse sintoma, o bloqueio peridural ou epidural apresenta-se como uma alternativa à procedimentos cirúrgicos e sua indicação está relacionada, principalmente, à resistência de tratamentos convencionais, como altas doses de opióides orais, transdérmicos ou sistêmicos. Assim, os resultados dessa revisão bibliográfica apontam a intervenção clínica de interrupção de estímulos nociceptivos por via peridural como método seguro, de bom custo-efetivo e com efeito analgésico satisfatório, o que implica uma opção considerável e eficaz para o controle da dor crônica. Por esse motivo, justifica-se a necessidade de continuidade de estudos científicos da técnica, visando sua evolução, redução dos custos e, sobretudo, aumento da eficácia clínica terapêutica.

Palavras-chave: Dor crônica, Anestesia epidural, Bloqueio neuromuscular.

Absract: This article aims to present clinical efficacy and the safety of anestesic peridural block at as interventional treatment in chronic pain control. The chronic pain is a recorrent and difficult to control symptom in several clinical entities with complex pathophysiological mecanisms that create social, fisical and mental importants repercussions to the population, besides implying hight costs in health service and to economy. Among the options of intervention and symptom control, the peridural or epidural block presents as an alternative to cirurgical procedures and the indication is related to resistance to conventional treatment, as hight oral, transdermic or sistemic opyoids doses. Thereby, the results of this review article emphasize the

\footnotetext{
${ }^{1}$ Centro Universitário de Caratinga (UNEC), Caratinga - MG. *E-mail: adrianasimoes.f@hotmail.com

2 Universidade Federal de Juiz de Fora (UFJF), Juiz de Fora - MG.

${ }^{3}$ Faculdade de Minas (FAMINAS BH), Belo Horizonte - MG.

4 Universidade Estadual de Mato Grosso do Sul (UEMS), Campo Grande - MS.

5 Universidade Vila Velha (UVV), Vila Velha - ES.

${ }^{6}$ Universidade Nove de Julho (UNINOVE), Bauru - SP.

7 Faculdade Dinâmica do Vale do Piranga (FADIP), Ponte Nova - MG.

${ }^{8}$ Centro Universitário Redentor (UniRedentor), Itaperuna - RJ.
} 
clinical intervention to interrupt nociceptive stimulation by peridural block, as a safe method, with good costeffective and satisfatory analgesic effect, that implies in a considerable and effective option to chronic pain control. Therefore, it's necessary ongoing cientific studies of this technique, aiming for improvement, reduce costs and increased therapeutic clinical efficacy.

Keywords: Chronic pain, Anesthesia epidural, Neuromuscular blockade.

Resumen: Este artículo buscó señalar la eficacia clínica y la seguridad del tratamiento intervencionista de los bloqueos anestésicos peridurales en el control del dolor crónico. El dolor crónico es un síntoma recurrente y de difícil control en diversas entidades clínicas con mecanismos fisiopatológicos complejos y que genera repercusiones sociales, físicas y mentales importantes para la población, además de resultar altos costos, tanto para el sistema de salud, como para la economía. Entre las formas de intervención y control de estos síntomas, el bloqueo peridural o epidural se presenta como una alternativa a los procedimientos quirúrgicos y su indicación está relacionada principalmente a la resistencia de tratamientos convencionales, como dosis altas de opioides orales, transdérmicos o sistémicos. Entonces el resultado de esta revisión bibliográfica señala la intervención clínica de interrupción de estímulo nociceptivo por la via epidural, como método seguro, buen costo efectivo y con efecto analgésico satisfactorio, lo que resulta una opción considerable y eficaz para el control del dolor crónico. Por este motivo se justifica la necesidad de continuidad de estudios científicos de la técnica, supervisando su evolución, reducción de costos y, sobre todo, aumento de su eficacia clínica terapéutica.

Palabras clave: Dolor crónico, Anestesia epidural, Bloqueo neuromuscular.

\section{INTRODUÇÃO}

A dor é uma manifestação clínica de diversas etiologias que, geralmente, é caracterizada como uma sensação desagradável, que pode estar associada ou não a uma lesão física ou, ainda, psicológica e, por isso, pode ser descrito como um possível indicador de surgimento de algum dano tecidual (HYLANDS- WHITE N, et al., 2017; VASCONCELOS FH e ARAÚJO GC, 2018).

Pode ser classificada em aguda e crônica de acordo com a sua duração, sendo a primeira, permanecendo até 3 meses do seu início e, a segunda, com duração superior a 3 meses desde o seu aparecimento. Além disso, possuem relevância clínica diversa, em que a dor aguda retrata uma possível lesão do organismo, com duração determinada e cicatrização do órgão lesado, enquanto que a dor crônica reflete um estado de hiperexcitabilidade neuronal, de caráter persistente, que mobiliza diversos locais do sistema nervoso central e periférico em resposta ao excesso de estímulos (VASCONCELOS FH e ARAÚJO GC, 2018; KRAYCHETE DC, 2016).

A dor crônica se configura como um fenômeno complexo, gerando impactos sociais, físicos e mentais, além de acarretar altos custos tanto para os sistemas de saúde, quanto para a economia (SOUZA I, 2017). Ainda assim, apesar de afetar negativamente a qualidade de vida, poucos são os estudos epidemiológicos acerca desse assunto. Estima-se que a sua prevalência no Brasil varia entre 29,7\% e 62,2\%, atribuindo essa variação ao perfil da população, ao tipo de estudo e à metodologia utilizada (SANTOS FAA, et al., 2015).

Ainda em seus estudos, Santos FAA, et al. (2015) descreve que a ocorrência da dor crônica é crescente quando relacionada ao sexo feminino, ao envelhecimento e a menores níveis socioeconômicos. Outrora, se atenua quando associada à prática regular de atividades físicas, empregos formais, níveis de escolaridade ascendentes e à condição socioeconômica favorável.

Por ser um quadro clínico frequente e resultar em tamanha incapacidade, para o controle da dor, podem ser adotados programas multidisciplinares baseados no modelo biopsicossocial, que englobam não só os procedimentos físicos, como também as respostas afetivas e fatores ambientais, proporcionando 0 contentamento do paciente e adesão ao tratamento (HYLANDS- WHITE N, et al., 2017). 
Uma das formas do controle da dor crônica, baseado em procedimentos físicos, é o bloqueio peridural que consiste na administração de medicações no espaço peridural, por meio de um cateter tunelizado que se localiza no espaço compreendido entre a dura-máter e a coluna vertebral, de forma a interromper a sensibilidade dolorosa, possibilitando que os pacientes refratários a outros tipos de tratamento e com limitações, possam ter o melhor manejo da dor (VITERI-TORO MM, et al., 2017; COCICOV AF, et al., 2004).

As infiltrações epidurais de esteroide é um termo geral que pode configurar uma diversidade de técnicas como abordagem por punção lombar (tanto mediana como paramediana) ou sacral. A técnica mais aplicada é pela punção lombar em função de uma menor variação anatômica e maior facilidade para infiltrar a solução próximo ao local de dor ou de lesão (NUNES RCS, et al., 2016).

Os medicamentos mais utilizados é o uso conjunto de opióides com anestésicos locais, mas também podem ser administrados anti-inflamatórios esteroidais, nos casos de processos inflamatórios associados (FRANÇA M, et al., 2015; NUNES RCS, et al., 2016; PATEL VB, et al., 2015, MANCHIKANTI L, et al., 2012).

Diante do exposto, o presente artigo tem como objetivo ratificar, através de uma revisão narrativa, a eficácia clínica e a segurança do tratamento intervencionista utilizando bloqueio anestésico peridural em pacientes com dor crônica.

\section{REVISÃO BIBLIOGRÁFICA}

A dor pode ser definida como uma experiência de caráter emotivo desagradável somado a uma vivência sensorial maçante e a uma lesão já estabelecida ou possível lesão (KLAUMANN PR, et al., 2008). O termo nocicepção está relacionado com o reconhecimento de sinais dolorosos pelo sistema nervoso, que geram informações acerca da lesão (KLAUMANN PR, et al., 2008).

A dor pode ser classificada de diversas formas: a fisiológica trata-se de um reflexo protetor do organismo para evitar uma injúria ou dano tecidual; já a patológica é utilizada uma vez instalado a injúria. Além disso, a dor pode seguir uma classificação temporal, sendo dividida em dor aguda ou crônica, sendo esta com duração superior a 3 meses desde o seu início (KLAUMANN PR, et al., 2008; HYLANDS- WHITE N, et al., 2017).

A dor aguda é um sintoma de alerta, reflete em aprendizado para não repetibilidade a fim de gerar ensinamentos de sobrevivência e está relacionada com um ponto de partida - lesão/injúria - e um ponto final - cicatrização; este tipo de dor está relacionada à preservação da integridade do indivíduo (KRAYCHETE DC, 2016; VASCONCELOS FH e ARAÚJO GC, 2018).

Em contrapartida, a dor crônica é considerada não adaptativa e está relacionada a um excesso de estímulo em diferentes locais do sistema nervoso central e periférico com hiperexcitabilidade neuronal (KRAYCHETE DC, 2016).

A dor crônica está entre as demandas médicas mais comuns e pode causar afastamentos laborais, incapacidades temporárias e permanentes além de elevada morbidade, sendo, portanto, considerada um problema de saúde pública (DE CASTRO S, et al., 2019; VASCONCELOS FH e ARAÚJO GC, 2018). Entende-se que, dessa forma, a dor crônica consiste num importante problema de gestão pública, trazendo impactos não apenas para o indivíduo acometido, mas também às famílias, aos sistemas de saúde e para a economia, em especial devido ao absenteísmo, aposentadoria precoce e perda de emprego (SOUZA I, 2017).

Alguns autores correlacionam dor crônica difusa no adulto com história anterior de hospitalização por acidentes e traumas psicológicos (na vida adulta ou infância), independentemente da classe social ou comorbidade psiquiátrica. Indica que o trauma também é um preditor de dor física, portanto, para toda dor, há um componente fisiológico e emocional de excitação ou sobrecarga de neurônios. Entre os pacientes com dor crônica, a prevalência de doença mental é alta, como ansiedade ou depressão. (KRAYCHETE DC, 2016).

Entende-se que a dor crônica pode, também, ser chamada de dor mal adaptativa, segundo a Sociedade Americana de Anestesiologistas, e pode ser definida quanto aos seus mecanismos subjacentes como mostrado no Quadro 1. 
Quadro 1 - Caracterização Mecanicista da Dor.

\begin{tabular}{|c|c|c|}
\hline Nociceptiva periférica & Neuropática periférica & $\begin{array}{c}\text { Neuropática central (“dor } \\
\text { centralizada") }\end{array}$ \\
\hline $\begin{array}{l}\text { - Inflamação ou danos mecânicos } \\
\text { aos tecidos. }\end{array}$ & $\begin{array}{l}\text { - Lesão ou disfunção dos nervos } \\
\text { periféricos. }\end{array}$ & $\begin{array}{l}\text { - Distúrbio central no } \\
\text { processamento da dor. }\end{array}$ \\
\hline - Responde a opióides e AINES. & $\begin{array}{l}\text { - Responde às terapias } \\
\text { farmacológicas de ação periférica }\end{array}$ & $\begin{array}{l}\text { - Responsivo a compostos } \\
\text { neuroativos que alteram os níveis }\end{array}$ \\
\hline - Responde a procedimentos. & e central. & $\begin{array}{l}\text { de neurotransmissores } \\
\text { envolvidos na transmissão da dor. }\end{array}$ \\
\hline $\begin{array}{l}\text { Exemplos: } \\
\begin{array}{l}\text { - Artrite Reumatóide } \\
\text { - Dor Oncológica } \\
\text { - Osteoartrite }\end{array}\end{array}$ & $\begin{array}{l}\text { Exemplos: } \\
\text { - Dor neuropática diabética } \\
\text { - Neuralgia pós-herpética }\end{array}$ & $\begin{array}{l}\text { Exemplos: } \\
\begin{array}{ll}\text { - } & \text { Fibromialgia } \\
\text { - Síndrome do intestino } & \text { irritável } \\
\text { - } & \text { Disfunção } \\
& \text { temporomandibular } \\
\text { - Cefaléia Tensional }\end{array}\end{array}$ \\
\hline
\end{tabular}

Fonte: Ferreira AS, et al., 2020. Adaptado de Clauw DJ, 2015.

A maior parte das síndromes que resultam em dor crônica (até as que persistem após uma lesão inflamatória aguda) desenvolvem-se através de um processo sensitivo anormal e através da indução de neuroplasticidade nas vias periféricas e centrais da dor (TREEDE RD, 2016).

De acordo com o estudo de Turk DC, et al., (2008), foram identificados os aspectos da vida cotidiana que indivíduos com dor crônica consideram importantes para avaliar as consequências de sua condição que estão representados na Tabela 1, sendo que $\mathrm{N}$ é o espaço amostral de pacientes com dor crônica que relataram tal patologia; os números fora dos parênteses tratam-se de uma classificação de escala de dor de 0 a 10 , sendo que 0 é a dor mínima e 10, a máxima; e os números entre parênteses, representa o desvio padrão.

Percebeu-se que as perspectivas dos pacientes são consideradas essenciais para entender o impacto de uma condição médica em suas vidas. Ao todo, foram encontrados 19 aspectos diferentes da vida diária que as pessoas estão sendo tratadas para diversas formas de dor crônica que se acreditam serem impactos por conta dos sintomas de dor cotidiana. Dessa forma, é possível perceber que é necessário tratar o paciente de forma holística, e não apenas a dor.

A prevalência de dor crônica ainda é pouco conhecida e estudada. Os estudos epidemiológicos sobre dor crônica são maiores em países desenvolvidos quando comparados a países subdesenvolvidos. No caso do Brasil, estudos apontam que a dor crônica é a principal causa de demanda no atendimento ambulatorial da dor (DE CASTRO S, et al., 2019). No que diz respeito à população geral, pode variar entre $11,5 \%$ a $55,2 \%$, apontam estudos (CIPRIANO A, et al., 2011). Porém, segundo a International Association for the Study of Pain (IASP), o valor da prevalência média é de 35,5\%.

Um fator importante para o aumento da prevalência de doenças crônicas é o envelhecimento da população que acarreta uma maior incidência de dor e incapacidade. Em muitos casos, a dor crônica é a queixa principal, e esta interfere de modo bem significativo na qualidade de vida do paciente. Estudos recentes apontam que a prevalência de dor crônica em idosos variam entre $29,7 \%$ e $52,8 \%$ e, por sua vez, ocorre com mais frequência nos membros inferiores (34,5\%) e região lombar (29,5\%) (MOURA CC, et al., 2017). 
Tabela 1 - Classificações de Importância Média por Condição de Dor.

\begin{tabular}{|c|c|c|c|c|c|c|c|c|}
\hline \multirow{2}{*}{\multicolumn{2}{|c|}{ Item }} & Enxaqueca & $\begin{array}{l}\text { Osteo- } \\
\text { Artrite }\end{array}$ & $\begin{array}{c}\text { Artrite } \\
\text { reumatoide }\end{array}$ & Dor lombar & $\begin{array}{l}\text { Pescoço/ } \\
\text { ombro }\end{array}$ & Fibromialgia & Neuropatia \\
\hline & & $\mathrm{N}=190$ & $\mathrm{~N}=158$ & $N=46$ & $\mathrm{~N}=453$ & $\mathrm{~N}=382$ & $\mathrm{~N}=227$ & $\mathrm{~N}=320$ \\
\hline 1 & Começar a dormir & $7,9(2,61)$ & $7,8(2,57)$ & $7,8(2,57)$ & $8,0(2,7)$ & $7,8(2,72)$ & $8,3(2,66)$ & $8,0(2,71)$ \\
\hline 2 & $\begin{array}{c}\text { Continuar dormindo a } \\
\text { noite }\end{array}$ & $8,3(2,33)$ & $8,7(1,96)$ & $8,6(2,52)$ & $8,5(2,18)$ & $8,3(2,36)$ & $8,8(2,00)$ & $8,3(2,59)$ \\
\hline 3 & Vida sexual & $6,7(3,56)$ & $6,2(3,54)$ & $6,2(3,26)$ & $6,9(3,45)$ & $6,6(3,42)$ & $6,5(3,53)$ & $6,9(3,47)$ \\
\hline 4 & Cuidar da família & $7,3(3,27)$ & $6,4(3,54)$ & $6,1(3,77)$ & $7,5(3,20)$ & $7,0(3,43)$ & $7,3(3,29)$ & $7,1(3,44)$ \\
\hline 5 & Relações Interpessoais & $8,0(2,44)$ & $7,7(2,70)$ & $7,8(2,44)$ & $7,9(2,55)$ & $7,6(2,81)$ & $8,4(2,14)$ & $7,7(2,94)$ \\
\hline 6 & Relações com amigos & $7,4(2,57)$ & $7,0(2,66)$ & $6,9(2,61)$ & $7,3(2,66)$ & $7,2(2,73)$ & $8,0(2,31)$ & $7,3(2,86)$ \\
\hline 7 & Emprego & $7,9(2,77)$ & $7,2(3,45)$ & $7,9(3,09)$ & $7,6(3,24)$ & $7,8(3,00)$ & $8,0(2,94)$ & $7,3(3,54)$ \\
\hline 8 & Cuidados com a casa & $7,9(2,30)$ & $8,0(2,02)$ & $7,6(2,63)$ & $8,2(2,20)$ & $7,9(2,33)$ & $8,4(1,92)$ & $7,9(2,29)$ \\
\hline 9 & $\begin{array}{l}\text { Planejamento de } \\
\text { atividades }\end{array}$ & $7,2(2,66)$ & $7,2(2,86)$ & $6,3(3,09)$ & $7,2(2,74)$ & $7,0(2,83)$ & $7,3(2,59)$ & $7,1(3,01)$ \\
\hline 10 & $\begin{array}{c}\text { Participação em eventos } \\
\text { e atividades familiares }\end{array}$ & $7,9(2,52)$ & $7,8(2,41)$ & $7,2(2,70)$ & $7,8(2,63)$ & $7,6(2,74)$ & $8,3(2,26)$ & $7,8(2,74)$ \\
\hline 11 & $\begin{array}{l}\text { Participação em } \\
\text { atividades sociais e } \\
\text { recreação }\end{array}$ & $7,7(2,67)$ & $7,8(2,40)$ & $6,6(3,12)$ & $7,9(2,47)$ & $7,7(2,63)$ & $8,1(2,41)$ & $7,7(2,64)$ \\
\hline 12 & Atividade Física & $8,2(2,48)$ & $8,7(2,12)$ & $8,0(2,57)$ & $8,7(2,06)$ & $8,4(2,27)$ & $8,6(2,06)$ & $8,5(2,48)$ \\
\hline 13 & Passatempos & $6,9(2,97)$ & $7,3(2,67)$ & $6,6(2,95)$ & $7,2(2,80)$ & $6,9(3,01)$ & $7,5(2,48)$ & $7,1(3,00)$ \\
\hline 14 & Aproveitamento da vida & $9,0(1,80)$ & $8,8(1,88)$ & $8,0(2,74)$ & $9,0(1,95)$ & $8,7(2,22)$ & $9,0(1,93)$ & $8,8(2,14)$ \\
\hline 15 & Bem- estar emocional & $8,8(2,03)$ & $8,7(1,90)$ & $8,0(2,37)$ & $8,7(2,21)$ & $8,5(2,36)$ & $8,9(2,03)$ & $8,7(2,28)$ \\
\hline 16 & Fadiga & $9,2(1,29)$ & $8,8(1,95)$ & $8,7(1,96)$ & $8,9(1,87)$ & $8,8(1,97)$ & $9,1(1,70)$ & $8,7(2,18)$ \\
\hline 17 & Fraqueza & $8,7(1,97)$ & $8,3(2,27)$ & $8,2(2,39)$ & $8,5(2,23)$ & $8,3(2,42)$ & $8,5(2,27)$ & $8,4(2,47)$ \\
\hline 18 & $\begin{array}{l}\text { Dificuldade de } \\
\text { Concentração }\end{array}$ & $8,6(2,01)$ & $8,0(2,45)$ & $7,8(2,23)$ & $8,0(2,64)$ & $8,1(2,55)$ & $8,5(2,25)$ & $8,1(2,66)$ \\
\hline 19 & Dificuldade de memória & $8,2(2,52)$ & $7,2(3,03)$ & $7,2(3,02)$ & $7,6(3,05)$ & $7,7(2,90)$ & $8,4(2,40)$ & $7,9(3,03)$ \\
\hline
\end{tabular}

Fonte: Ferreira AS, et al., 2020. Adaptado de Turk DC, et al., 2008. 
Um estudo feito por Cipriano A, et al. (2011) mostrou que a maior incidência de causa de dor crônica é a lombalgia, seguida por dores nos membros inferiores e, em terceiro lugar, a cervicalgia (Tabela 2).

Tabela 2 - Localização da dor crônica informada pelos pacientes.

\begin{tabular}{lcc}
\hline & \multicolumn{2}{c}{ Total } \\
\cline { 2 - 3 } Categorias & $\mathbf{N}$ & $(\%)$ \\
\hline Lombalgia & 24 & $(21,1 \%)$ \\
Membros Inferiores & 18 & $(15,8 \%)$ \\
Cervicalgia & 15 & $(13,2 \%)$ \\
Membros Superiores & 14 & $(12,3 \%)$ \\
Dor generalizada & 11 & $(9,6 \%)$ \\
Cefaleia & 8 & $(7,0 \%)$ \\
Dor orofacial & 6 & $(5,3 \%)$ \\
Outras & 18 & $(15,8 \%)$ \\
\hline
\end{tabular}

Legenda: ${ }^{* \star}$ Alguns pacientes apresentaram dor em mais de uma localização.

Fonte: Ferreira AS, et al., 2020. Adaptado de Cipriano A, et al., 2011.

Quanto ao tratamento das inúmeras causas de dor crônica supracitadas, percebe-se que por meio das técnicas intervencionistas analgésicas, pode-se ofertar uma melhora tanto na qualidade de vida, quanto no controle da dor e até redução na dose de analgésicos (orais, transdérmicos ou intravenosos) naqueles pacientes refratários aos tratamentos convencionais ou que ficaram limitados aos efeitos colaterais intoleráveis (VITERI - TORO MM, et al., 2017).

Segundo a Sociedade Americana de Geriatria, a dor crônica pode ser tratada levando como base a Escada Analgésica da Organização Mundial de Saúde (OMS), criada em 1996, que teve, na época, o intuito de tratar apenas dor oncológica. O primeiro degrau, o tratamento da dor leve, recomenda-se os anti-inflamatórios nãohormonais (AINES) e os analgésicos comuns, como a dipirona e o paracetamol. Para o tratamento das dores neuropáticas, as medicações adjuvantes, como antidepressivos e anticonvulsivantes, são também recomendadas; O segundo degrau é utilizado para o tratamento de dores leves a moderadas, e inclui a adição de um opióide fraco, quando falhou a prescrição anterior. A Codeína e o Tramadol estão disponíveis no Brasil; O terceiro degrau substitui o opióide fraco por um forte, que no Brasil inclui a Morfina, a Metadona, a Oxicodona, a Hidromorfona, o Fentanil transdérmico e a Buprenorfina transdérmica, recomendado para o controle de dores moderadas a graves que não são aliviadas com a prescrição do segundo degrau (AMERICAN GERIATRICS SOCIETY, 2015).

A analgesia neuroaxial (Intratecal ou peridural) ou periférica, compõe o quarto passo da escada analgésica e possui indicação quando houver resistência a altas doses de opióides orais, transdérmicos ou sistêmicos; dor sensível aos opióides sistêmicos, mas acompanhado por efeitos colaterais intoleráveis; dor que não pode ser tratada com outras modalidades de intervenção; e em pacientes com sobrevida menor do que 3 meses (VITERI - TORO MM, et al., 2015).

Coincidindo com as principais causas de dor crônica, as clínicas de lombalgias e lombociatalgias são, também, as causas mais comuns para necessidade de infiltração epidural, elas possuem etiologia mecânicodegenerativas e causas não mecânicas (metabólicas, psicossomáticas), o que pode justificar a busca de um especialista em dor pelos pacientes (NUNES RCS, et al., 2016; PATEL VB, et al., 2015; MANCHIKANTI L, et al., 2012).

Assim, injeção peridural ou epidural vem sendo utilizadas há décadas como uma alternativa a procedimentos cirúrgicos, atualmente sendo orientado por método fluoroscópico ou tomográfico, mas também havendo técnica para injeções "às cegas", como é o caso da injeção epidural interlaminar lombar, contudo, essa técnica, apesar de ser a mais comum, não é a que apresenta melhores resultados (NUNES RCS, et al., 2016; PATEL VB, et al., 2015; MANCHIKANTI L, et al., 2012). 
A administração peridural de analgésicos consiste em três vias que são a interlaminar (IL), a caudal e a transforaminal (TF) (ZARGAR S, et al., 2019). As mais utilizadas pelos profissionais anestesistas são a caudal e a tradicional IL. A abordagem da IL, por meio da técnica da linha média ou paramediana, obtém o acesso ao espaço peridural através do ligamento amarelo, avança entre as camadas da coluna vertebral para o espaço peridural posterior com a técnica de perda de resistência. É indicada especificamente no tratamento da dor radicular que acomete as regiões lombar, torácica e cervical da coluna vertebral (GEGEL BT, et al., 2019).

Sendo uma das mais antigas, a abordagem caudal é feita acessando por meio de uma agulha o espaço peridural através do hiato sacral. Este, por sua vez, é recoberto pelo cruzamento das fibras dos ligamentos sacrococcígeos superficial e profundo e oblitera a extremidade inferior do espaço epidural sendo, portando, um fator determinante no sucesso e segurança da técnica pela via caudal, por meio da detecção de variações anatômicas nessa localização. Com o avançar da idade esses ligamentos tornam-se mais espessos, o que torna um desafio para o médico na identificação das margens do hiato e predispõe riscos ao procedimento. Esta técnica é amplamente usada para anestesia cirúrgica em crianças e em adultos no tratamento da dor crônica (GEGEL BT, et al., 2019; KAO SC e LIN CS, 2017; NAJMAN IE, et al., 2011).

Já a abordagem transforaminal, consegue o acesso ao espaço peridural anterior através do caminho percorrido pela agulha no forame intervertebral. Está voltada no gerenciamento avançado e intervencionista da dor, tendo a vantagem teórica de poder conduzir a substância até o local de origem da dor no espaço peridural anterior. Além disso, é muito utilizada nas áreas sacral e lombar da coluna vertebral (GEGEL BT, et al., 2019).

Na prevenção de complicações como as relacionadas a abordagem (caudal, IL ou TF) ou ao traumatismo da agulha, o avanço da tecnologia proporcionou o uso de meios de imagem como a fluoroscopia e o ultrassom em conjunto aos acessos peridurais sendo, hoje, fundamental para a realização desse procedimento (GEGEL BT, et al., 2019; KAO SC e LIN CS, 2017).

A fluoroscopia é definida como o padrão ouro para algumas vias de administração da injeção peridural, porém, pelo seu alto custo, por expor o paciente à radiação e pela necessidade de se ter um espaço especial, a ultrassonografia é a alternativa excelente quando a fluoroscopia não estiver disponível (KAO SC e LIN CS, 2017).

Referente aos medicamentos usados, é frequente o uso em conjunto de opióides com os anestésicos locais, tendo como finalidade um melhor bloqueio, uma redução na necessidade anestésica e diminuição do bloqueio motor. Contudo, em se tratando de lombalgias, e por suas causas serem amplas e diversas, porém, parcialmente vinculada a processos inflamatórios, a injeção de anti-inflamatórios esteroidais é um procedimento coerente, e pode ser associado ou não a anestésicos locais. Contudo, novas evidências mostram que a aplicação unicamente com anestésicos locais pode ser igualmente eficiente; em contraposição, há outros estudos que mostram que os esteróides parecem indicar uma potencial superioridade em um longo prazo (FRANÇA M, et al., 2015; NUNES RCS, et al., 2016; PATEL VB, et al., 2015; MANCHIKANTI L, et al., 2012).

\section{CONSIDERAÇÕES FINAIS}

Por meio da análise bibliográfica deste estudo pode-se concluir que a interrupção de estímulos nociceptivos em sua origem ou próximo dela, por via peridural, é uma intervenção que permite o aumento do efeito analgésico e a redução dos efeitos colaterais, proporcionando uma melhora na qualidade de vida do paciente. Compreende um tratamento intervencionista minimamente invasivo, que possui uma boa relação custo-efetividade e que foi importante na redução das indicações cirúrgicas. Além disso, mostrou-se ser uma técnica com desejável nível de segurança e tolerabilidade, o que aumenta as chances de êxito na adesão ao tratamento pelo paciente e, consequentemente, o controle da dor crônica. Por esse motivo, justifica-se a necessidade de continuidade de estudos científicos da técnica, visando sua evolução, redução dos custos e, sobretudo, aumento da eficácia clínica terapêutica. 


\section{REFERÊNCIAS}

1. ALVES TCA, et al. Bloqueio peridural com corticoide em hérnia discal. Revista Neurociências, $2015 ; 23$ (4): 575-580.

2. AMERICAN GERIATRICS SOCIETY. American Geriatrics Society 2015 Updated Beers Criteria for Potentially Inappropriate Medication Use in Older Adults. J Am Geriatr Soc., 2015; 63 (11): 2227-46.

3. BARRETO EPS, et al. Bloqueio neurolítico da cadeia simpática lombar melhora dor crônica em paciente portadora de isquemia crítica de membro inferior. Rev. Bras. Anestesiol., 2018; 68 (1).

4. BEYAZ SG. Comparação das técnicas transforaminal e interlaminar de injeções epidurais de esteróides para o tratamento de dor lombar crônica. Brazilian Journal of Anesthesiology, 2017; 67 (1): 21-27.

5. CIPRIANO A, et al. Perfil do paciente com dor crônica atendido em um ambulatório de dor de uma grande cidade do sul do Brasil. Rev Dor. São Paulo, 2011; 12 (4): 297-300.

6. CLAUW DJ. Diagnosing and treating chronic musculoskeletal pain based on the underlying mechanism(s). Revista Best Practice \& Research Clinical Rheumatology, 2015; 29: 6-19.

7. COCICOV AF, et al. Uso de corticosteróides por via peridural nas síndromes dolorosas lombares. Revista Brasileira de Anestesiologia, 2004; 54 (1): 129-141.

8. DE CASTRO S, et al. Implementação de atendimento ambulatorial para dor crônica: resultados preliminares. Revista Brasileira de Anestesiologia, 2019.

9. FRANÇA MA, et al. Anestesia peridural: vantagens e desvantagens na prática anestésica atual. Rev Med Minas Gerais, 2015; 25 (4): 36-47.

10. HART D, et al. A Review of the Analgesic Benefits and Potential Complications Related to Epidural Corticosteroid Injections. AANA jornal, 2019; 87: 71-79.

11. HYLANDS-WHITE N, et al. An overview of treatment approaches for chronic pain management. Rheumatology international, 2017; 37 (1): 29-42.

12. NAJMAN IE, et al. Bloqueio peridural caudal: técnica anestésica de uso exclusivo em crianças? É possível sua realização em adultos? Qual o papel do ultrassom nesse contexto?. Rev. Bras. Anestesiol, 2011; 16 (1).

13. KAO SC, LIN CS. Bloqueio epidural caudal: uma revisão atualizada de anatomia e técnicas. BioMed Research International, 2017.

14. KLAUMANN PR, et al. Patofisiologia da dor. Arquivos de Ciência Veterinária, 2008; 13 (1): 11532.

15. KRAYCHETE DC. A escuta clínica do paciente com dor e sua importância no cotidiano do médico. Revista Dor, 2016; 17(4): 243-244.

16. KRELING MC, et al. Prevalência de dor crônica em adultos. Rev. bras. enferm., 2006; 59 (4).

17. MANCHIKANTI L, et al. O papel das injeções peridurais interlaminar fluoroscópicas no controle da dor crônica da hérnia ou radiculite do disco lombar: um estudo randomizado, duplo-cego. Pain Practice, $2012 ; 13$ (7): 547-558.

18. MOURA CC, et al. Impactos da dor crônica na vida das pessoas e a assistência de enfermagem no processo. Av Enferm. 2017; 35 (1): 53-62.

19. NUNES RCS, et al. Avaliação do bloqueio epidural como terapêutica em pacientes com ciatalgia secundária a herniação discal lombar. Rev. bras. ortop., São Paulo, 2016; 51 (4): 424-430.

20. OMS. Cancer pain relief - with a guide to opioid availability, 1996; $2^{\underline{a}}$ Edição.

21. PATEL VB, et al. Terapias intervencionistas para lombalgia crônica: uma revisão focada (eficácia e resultados). Anestesiologia e Medicina da Dor, 2015; 5 (4).

22. SANTOS FAA, et al. Prevalência de dor crônica e sua associação com a situação sociodemográfica e atividade física no lazer em idosos de Florianópolis, Santa Catarina: estudo de base populacional. Rev. bras. epidemiol., São Paulo, 2015; 18 (1): 234-247.

23. SOUZA I, et al. Resilience profile of patients with chronic pain. Cad. Saúde Pública, Rio de Janeiro, 2017 ; 33 (1).

24. TREEDE RD. Gain control mechanisms in the nociceptive system. Revista DOR, 2016; 157 (6): 1199-1204.

25. TURK DC, et al. Identifying important outcome domains for chronic pain clinical trials: An IMMPACT survey of people with pain. Rev. PAIN, 2008; 137 (2): 276-285.

26. VASCONCELOS FH, ARAUJO GC. Prevalence of chronic pain in Brazil: a descriptive study. BrJP, São Paulo, 2018; 1(2): 176-179.

27. VITERI-TORO MM, et al. Colocación de catéter peridural en el paciente de dolor y cuidado paliativo: revisión narrativa. Universitas Médica, 2017; 58 (4).

28. ZARGAR S, et al. Addition of dexmedetomidine and neostigmine to $1.5 \%$ lidocaine and triamcinolone for epidural block to reduce the duration of analgesia in patients suffering from chronic low back pain. J Med Life, 2019; 12(3):260265. 